\title{
Attention to self-referential stimuli: Can I ignore my own face?
}

\author{
Christel Devue and Serge Brédart \\ Department of Cognitive Science, University of Liège, Belgium
}

\begin{abstract}
Auto-referential materials (i.e., the own name) have been described as particularly prone to capture attention. Some recent studies have questioned this view and showed that these own name effects are temporary and appear only in specific conditions: when enough resources are available (Harris \& Pashler, 2004) or when the own name is presented within the focus of attention if it is a task-irrelevant stimulus (Gronau et al., 2003). In the present study, a stimulus that is unique to each individual was used: the self-face. In Experiment 1, the selfface produced a temporary distraction when presented at fixation during a digit-parity task. However, this distraction was not different from that triggered by another highly familiar face. In Experiment 2, the self-face failed to produce interference when presented outside the focus of attention. These results confirm recent findings showing that auto-referential materials do not automatically summon attention and have a distractive power only in specific conditions.
\end{abstract}

Keywords: face perception, familiarity, selective attention, self perception, visual attention. PsyclNFO classification: 2300 Human Experimental Psychology; 2323 Visual Perception; 2346 Attention.

Corresponding author:

Christel Devue

Department of Cognitive Science

University of Liège

Bd du Rectorat, 5 (Bat. B32)

B-4000 Liège

Belgium

Email: cdevue@ulg.ac.be

Tel.: +32[0]4 3662021

Fax : +32[0]4 3662859 


\section{INTRODUCTION}

Since the late fifties, self-referential stimuli have been described as particularly important with regard to their capacity to grab attention by comparison with other stimuli. This claim emerged from a famous study by Moray (1959). Using the method of shadowing during a dichotic listening task, Moray (1959) found that 4 participants out of $12(33 \%)$ remembered that they had heard their own name at its first presentation to the unattended ear while they were shadowing (i.e., repeating aloud) a message presented to the other ear. On the contrary, a short list of neutral words presented many times to the unattended ear showed no trace of being remembered. This suggested that some high-priority important stimuli can capture attention because of their meaning. However, there was no way to exclude the possibility that subjects from time to time shifted their attention to the to-be-ignored message (see Lachter, Forster, \& Ruthruff, 2004). More evidence for some "own name effects" has also been found in various studies using different procedures. For instance, in a visual analogy of the Moray's procedure, Wolford and Morrison (1980) showed that when instructed to make a parity judgment on two digits flanking a to-be-ignored word, a higher proportion of participants subsequently reported that they had seen their own name in comparison with words presented the same number of times during the experiment. Shapiro's team later showed that the own name is particularly resistant to the attentional blink (Shapiro, Caldwell, \& Sorensen, 1997) and to repetition blindness (Arnell, Shapiro, \& Sorensen, 1999). In addition, Mack and Rock (1998) found that almost all of their participants (88\%) detected their own name when presented under conditions of inattention in the inattentional blindness paradigm. By comparison, only $65 \%$ of participants detected another first name and $50 \%$ of participants detected very frequent words in the English language (e.g. "house"). Using a visual search task, Mack and Rock (1998) also showed that, contrary to other names, the own name pops out of a display of up to 12 items.

All these studies investigated the attentional properties of the own name. However, the name (i.e., the first name as well as the last name) is a property that we may share with other people. By contrast, the face is a unique self-referential characteristic (with the exception of twins) and hence constitutes a better way to investigate the specificity of self-referential stimuli. However, few studies (Brédart, Delchambre, \& Laureys, 2006; Laarni, Koljonen, Kuistio, Kyröläinen, Lempiäinen, \& Lepistö, 2000; Ninomiya, Onitsuka, Chen, Sato, \& Tashiro, 1998; Tong \& Nakayama, 1999) investigated the attentional properties of the selfface. Recently, using a face-name interference paradigm, Brédart et al. (2006) found that the self-face flanking a classmate's name produced a stronger interference on the processing of this name than in the reverse situation where a classmate's face flanked the participant's own name. This suggests that the self-face also has some attention-grabbing capacity. Tong and Nakayama (1999), in a visual search task, demonstrated that the self-face was more quickly detected among distractors than strangers' faces even when presented in atypical orientations and after hundreds of trials. Several ERP or PET studies also found specific electrophysiological and neuronal responses associated with attention to the self-face by comparison with other unfamiliar faces (Gray, Ambady, Lowenthal, \& Deldin, 2004; Ninomiya et al., 1998; Sugiura et al., 2000; Sui, Zhu, \& Han, 2006; Tanaka, Curran, Porterfield, \& Collins, 2006) or familiar faces (Sui et al., 2006). All these findings suggest that important stimuli may benefit from specific attention-grabbing capacities. Taken together, these results seem in favour of a late selection theory of attention (Deutsch \& Deutsch, 1963) because they suggest that the meaning and significance of some stimuli can determine their selection by attention. However, many studies used neutral words, unfamiliar faces or names as control stimuli. Hence, these results might just reflect a familiarity effect rather than a genuine "self-effect". That might weaken the argument in favour of a late 
selection theory of attention because this could indicate that familiarity rather than meaning determines the attentional selection.

In addition, recent research has seriously questioned the specificity of self-referential stimuli by suggesting that such stimuli are not really special and do not benefit from particular attention-grabbing capacities. A study by Bundesen, Killingsbæk, Houmann, and Jensen (1997) challenged previous findings with respect to the attention-grabbing capacities of the own name. They used displays composed of four names, two written in red (targets to be reported) and two written in white (distractors to be ignored). The participant's name appeared on some trials either as target either as distractor. Results showed that the own name did not cause more interference than other names when it was a distractor suggesting that it does not automatically grab attention. Laarni et al. (2000) found that when participants had to perform a matching task on two faces presented at foreground while ignoring the background composed of a matrix of faces among which the participant's own face or a celebrity face (the Finnish President) was presented on some trials (i.e., critical trials), only $18 \%$ of participants reported that they had seen their own face during the task and the performance was similar for both familiar faces. These results could be interpreted in terms of an early selection of attention occurring at an early stage of processing (Broadbent, 1958) preventing the processing of significant stimuli when they were already discarded from the perceptual process by the properties of their low level features (e.g., irrelevant colour, irrelevant background location, see also Bundesen et al., 1997).

Finally, some studies using the own name moderated findings presented here above by showing that some "self-effect" can occur but only when specific conditions are fulfilled. For instance, Kawahara and Yamada (2004) replicated Bundesen et al. (1997)'s findings but additionally showed, using an adapted version of the attentional blink paradigm, that the own name only attracted attention when participants were set to identify target names whereas it did not when participants were set to find a target colour. Similarly, using a Stroop-like task Gronau, Cohen, and Ben-Shakhar (2003) found that the own name attracted attention when it was presented centrally within the participant's attentional focus. However, when it was presented peripherally it attracted attention only when it was task relevant but not when it was task irrelevant. Harris, Pashler, and Coburn (2004) also call Mack and Rock (1998)'s visual search findings into question by showing that even if the participant's own name is detected more rapidly than other names, the search slopes they obtained were not flat enough to claim that the own name pops out. In another experiment based on the paradigm designed by Wolford and Morrison (1980), Harris and Pashler (2004) showed that the presentation of the own name can cause a distraction and slow down reaction times on a digit-parity task by comparison with neutral words on condition that enough capacity is available. In this case however the distraction is only present during the first occurrences of the own name and the response quickly habituates. Moreover, the own name did not show special attention grabbing property when display loads were more substantial. Taken together, this set of results suggests that the first occurrences of one's own name may provoke an involuntarily shift of attention when the perceptual load of one's ongoing activity is low and enough capacity is available for one's name to be perceived, but that it rapidly loses its attention grabbing character.

From this overview of the literature, it remains unclear whether or not self-referential materials have specific attention-grabbing capacity. Moreover, controls used to determine whether these stimuli have special attention-grabbing capacities were not always the most appropriate ones and some confounding factors such as familiarity, frequency of use or emotional valence could have interfered. The aim of the present study was to evaluate the distractive potency of an extremely distinctive self-significant stimulus, i.e. the self-face, and in the meantime disentangling the potential effect of stimulus familiarity. In Experiment 1, we tested whether one's own face is harder to ignore as a distractor than other unfamiliar faces. If 
results found in the Harris and Pashler (2004) study can be generalised from the own name to other self-significant stimuli, it was expected that the first presentation of the participants' face would produce a momentary distraction. To determine how much such effect was specific to one's own face, distraction due to the presentation of another personally familiar face (the face of a participant's classmate) was also evaluated.

In the Harris and Pashler (2004) study, distractors were presented centrally, i.e. between the items to be processed for the primary task. Gronau et al. (2003) demonstrated that the presentation of one's name outside the focus of attention did not interfere with a primary task if one's name was not relevant to this primary task. Hence, it was predicted that the presentation of the participant's own face as an irrelevant flanking distractor would produce no disruption of the primary task at all. This point was addressed in Experiment 2.

\section{EXPERIMENT 1}

The experiment used the digit-parity task described by Wolford and Morrison (1980) and Harris and Pashler (2004), i.e. a task in which participants indicated whether two simultaneously presented digits had the same parity or not. By analogy with the Harris and Pashler study, during the first block of trials, familiar faces (either one's face or a classmate's face) were presented infrequently. In the second block of trials familiar faces were presented on half of the trials.

\subsection{Method}

\subsubsection{Participants}

A total of 48 undergraduate students (mean age $=20 ; 30$ women) volunteered. They had all known their same-gender classmate for at least 2 years. Participants were recruited by pairs so that each participant served as the classmate for the other participant.

\subsubsection{Materials and stimuli}

Displays were presented on a monitor controlled by a PC computer. They consisted of two digits flanking a face and were viewed at a distance of $56 \mathrm{~cm}$ controlled by means of a chin rest. The digits subtended $0.7^{\circ}$ by $0.5^{\circ}$ of visual angle, were spaced $4^{\circ}$ apart, and located $2^{\circ}$ from fixation each. They were written in black against a grey background. All face stimuli were greyscales images of full-frontal views of faces without facial hair or glasses. The picture of the participant's own face was presented in a mirror-reversed orientation, i.e. the view in which we typically see our own face. The pictures were centred at fixation and subtended $4.1^{\circ}$ by $3.3^{\circ}$ of visual angle (see Fig. 1 b).

\subsubsection{Procedure}

The experiment consisted of two blocks of 48 trials each. In Block 1, the participant's own face and the face of the participant's classmate were presented once each. Half of the participants saw their own face on Trial 29 and their classmate's face on Trial 39, the other half saw these two familiar faces in the reverse order. Pictures of unfamiliar faces were shown on the other trials. Each unfamiliar face appeared once only. In Block 2, the participant's face appeared on 12 trials, the classmate's face appeared on 12 trials and 24 new unfamiliar faces appeared on the remaining 24 trials. Faces were presented randomly except that the same face could not be shown twice successively.

Participants were instructed to focus on the digit-parity task and ignore the faces. Each trial began with a fixation point (diameter $=0.2$ degrees) presented in the centre of fixation for $1 \mathrm{~s}$. The point was followed, after $500 \mathrm{~ms}$, by a 200-ms (unmasked) exposure of the digits and face. Half of the participants pressed the " $C$ " key of a computer keyboard if the digit parity 
matched, and otherwise pressed the " $\mathrm{N}$ " key. These keys were reversed for the other participants. A 1-s interval separated successive trials (see Fig.1a).

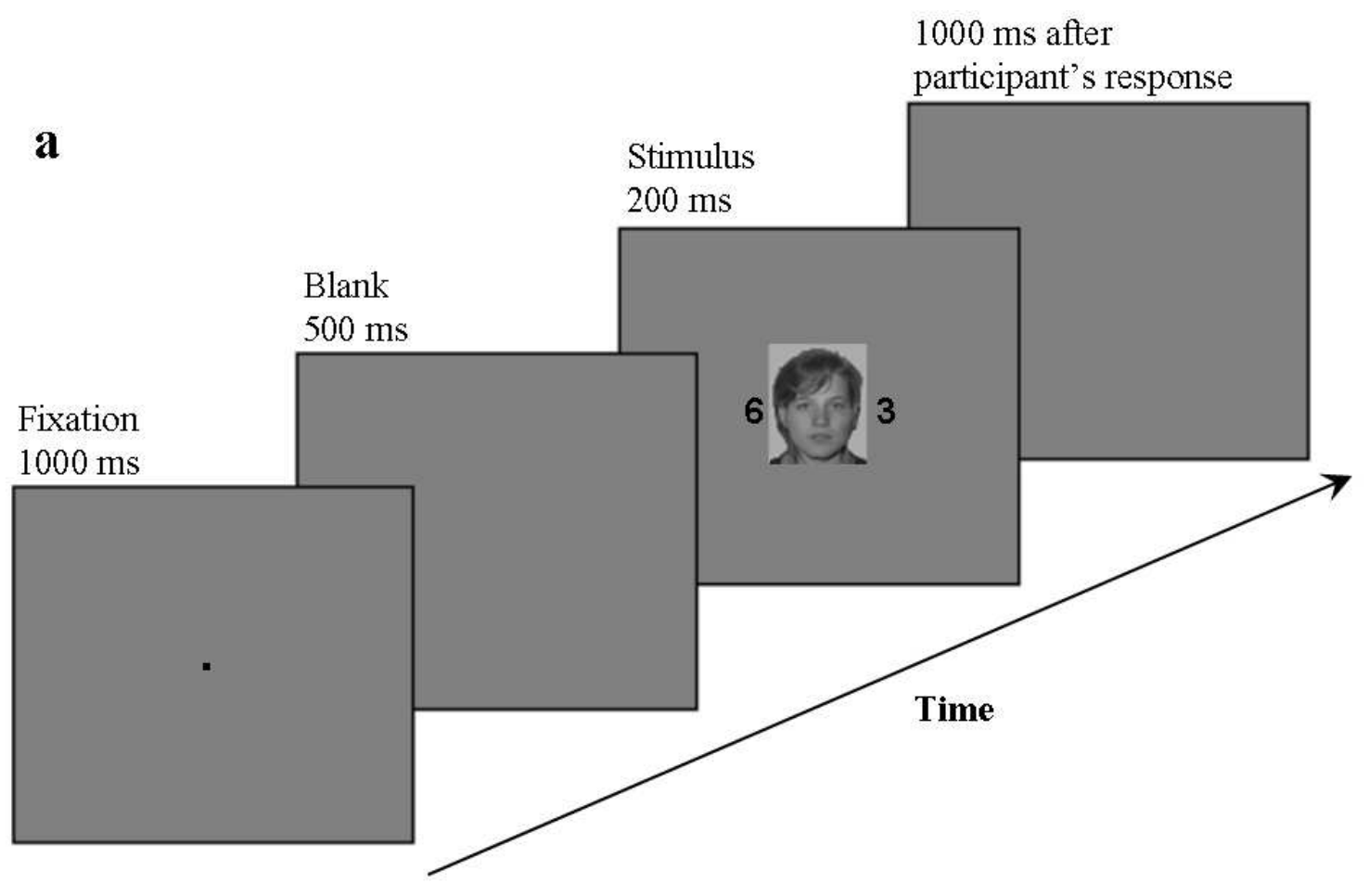

b

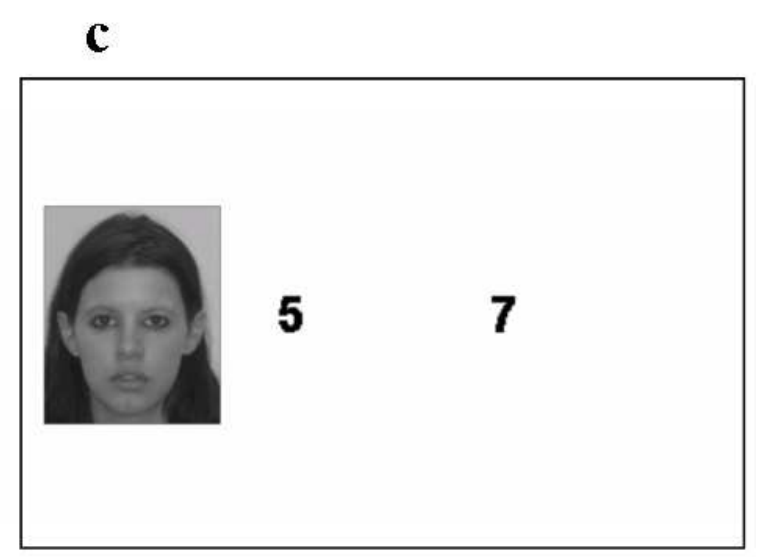

Figure 1. (a) Illustration of the time course of a single trial in the two experiments (the stimulus display represented here corresponds to displays in Experiment 1); (b) in Experiment 1, faces were presented at fixation between two digits; (c) in Experiment 2, faces were presented at periphery, randomly on the left or on the right (here on the left) of two digits.

\subsection{Results}

\subsubsection{Reactions times}

Data of 15 participants who made errors on Trial 29 or 39 were discarded.

Block 1. A mixed two-way 2 (Order: self-classmate, classmate -self) by 5 (Trial: 20 to 28, 29, 30 to $38,39,40$ to 48 ) ANOVA with repeated measures on the last factor was conducted on reaction times (RTs). There was a significant effect of Trial, $F(4,124)=8.32, p=0.00001$. HSD Tukey post-hoc indicated a significant elevation on the first two trials in which a 
familiar face was presented by comparison with preceding and following trials, all $p \mathrm{~s}<0.05$. There was no significant effect of Order, $F(1,31)<1$, and no significant interaction, $F(4,124)$ $<1$ (see Fig.2a).

Block 2. A one-way ANOVA with Identity (self, classmate, unfamiliar) as within-subject factor was conducted on RTs. There was a significant effect of Identity, $F(2,64)=3.28, p=$ 0.04. HSD Tukey post-hoc indicated that the difference between the RTs in the 'self-face' condition $(M=1070 \mathrm{~ms})$ and the RTs in the 'unfamiliar face' condition $(M=1002 \mathrm{~ms})$ was marginally significant, $p=0.058$. In addition, RTs when the classmate was presented $(M=$ $1062 \mathrm{~ms}$ ) tended to be slower than RTs when an unfamiliar face was presented, $\mathrm{p}=0.10$. Finally, there was no significant difference between the 'self-face' condition and the 'classmate's face' condition, $p=0.96$ (see Fig.2b). Data of Block 2 were then split in 2 parts to examine separately the pattern of performance on the first and second halves of trials (see Fig.2c). The analysis of RTs during the first half of Block 2 confirmed the above effect of the Identity, even if the difference was only marginally significant, $F(2,64)=2.99, p=0.058$. Conversely, the analysis of RTs during the second half of Block 2, did not reveal any effect of the Identity, $F(2,64)<1$.

\subsubsection{Error rates}

Data of one participant who misunderstood the instructions were discarded (this participant responded to 26 out of 96 trials only).

Block 1. A mixed two-way 2 (Order: self-classmate, classmate -self) by 5 (Trial: 20 to 28, 29, 30 to $38,39,40$ to 48 ) ANOVA with repeated measures on the last factor was conducted on error rates. There was a significant effect of Trial, $F(4,180)=4.97, p=0.0008$. HSD Tukey post-hoc indicated that when a familiar face was presented for the second time $(M=25.4 \%$ on trial 39), error rates were higher by comparison with preceding $(M=8.3 \%$ for trials 20 to 28 and $M=7.1 \%$ for trials 30 to 38) and following trials $(M=8.5 \%$ for trials 40 to 48$)$ and by comparison with trial $29(M=10.6 \%)$, all $p$ s $<0.02$. There was no significant effect of Order, $F(1,45)=1.22 ; p=0.27$, and no interaction, $F(4,180)<1$ (see Fig. $2 \mathrm{~d}$ ).

Block 2. A one-way ANOVA with Identity (self, classmate, unknown) as within-subject factor was conducted on error rates and showed no significant effect, $F(2,92)<1$ (see Fig.2e).

\subsection{Discussion}

Present results indicate that the self-face, like the own name, can produce a distraction. Like the own name however, the self-face has no enduring attention grabbing capacity as the response habituates after a few presentations. In addition, the pattern of interference produced by the self-face and by the other highly familiar face was similar both in Block 1 and Block 2 . The distraction produced by the two familiar faces impacted both dependant measures but was stronger on reaction times than on error rates. This suggests that the irrelevant presence of a familiar face mainly slows down the processing of the task-relevant items (i.e. the digits) but has a more limited effect on response accuracy. The second experiment examined the effect of the presentation of a familiar face (i.e., the self-face or the classmate's face) outside the focus of attention. It was predicted that the presentation of the self-face as an irrelevant flanking distractor would produce no disruption of the digit parity task at all (see Gronau et al., 2003). 

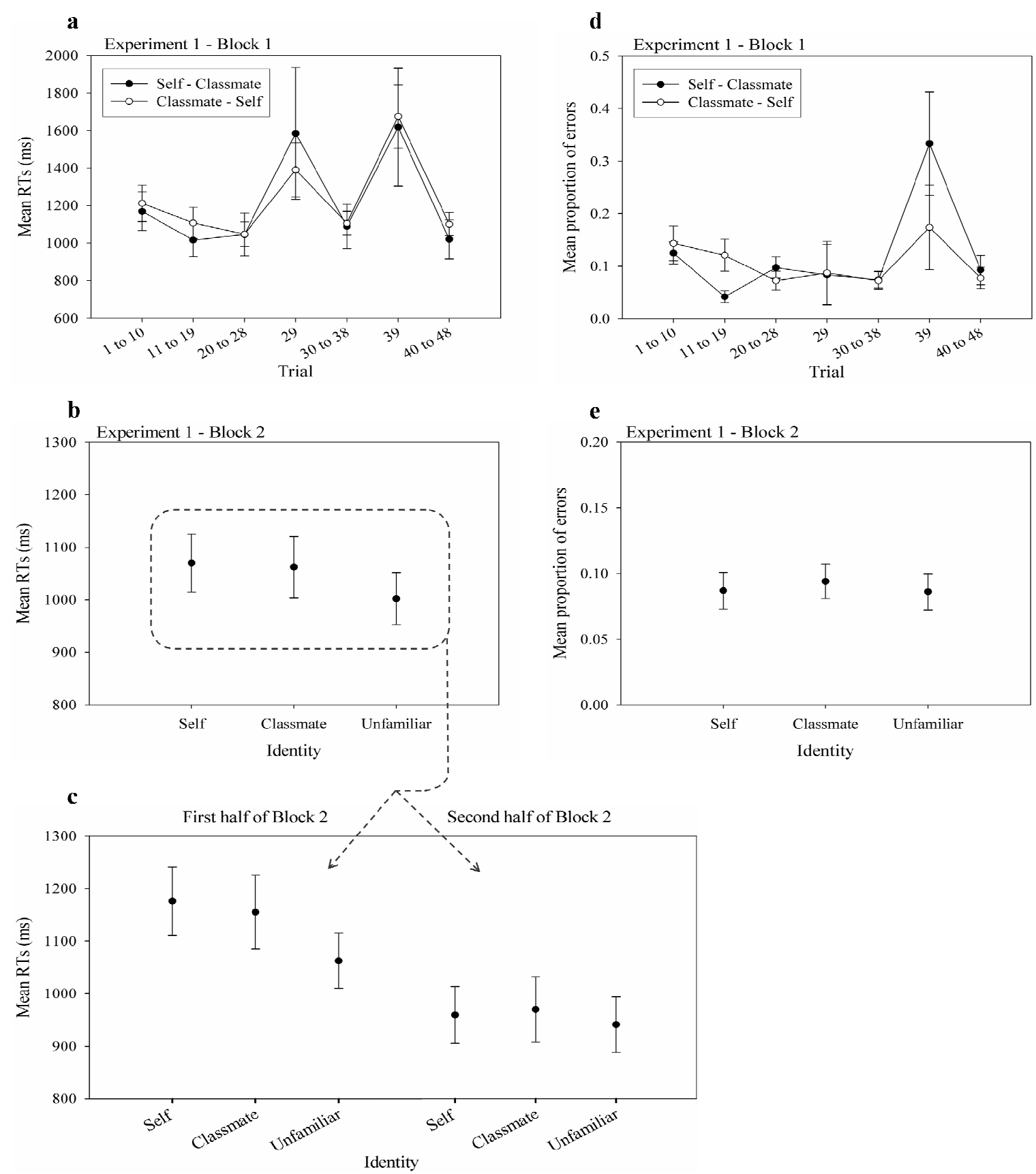

Figure 2. Results of Experiment 1: (a) Mean reaction times (RTs) and (d) mean error rates to the digit-parity task as a function of the order of presentation of the familiar faces in Block 1; familiar distractor faces appeared on trials 29 and 39. (b) Mean RTs and (e) mean error rates as a function of the Identity of the distractor face presented in Block 2. (c)Mean RTs as a function of the distractor face's Identity presented on the first and the second halves of Block 2. Error bars represent the standard error of the means.

\section{EXPERIMENT 2}

\subsection{Method}

\subsubsection{Participants}

A total of 48 undergraduate students (mean age $=21 ; 20$ women) volunteered. They had all known their same-gender classmate for at least 2 years. Participants were recruited by pairs so that each participant served as the classmate for the other participant. 


\subsubsection{Stimuli and procedure}

Experiment 2 was identical to Experiment 1 except that faces were presented in a flanking position instead of a central position. Faces were randomly presented on the left side of the left digit or on the right side of the right digit so that the centre of the picture was $5^{\circ}$ from fixation (see Fig.1c).
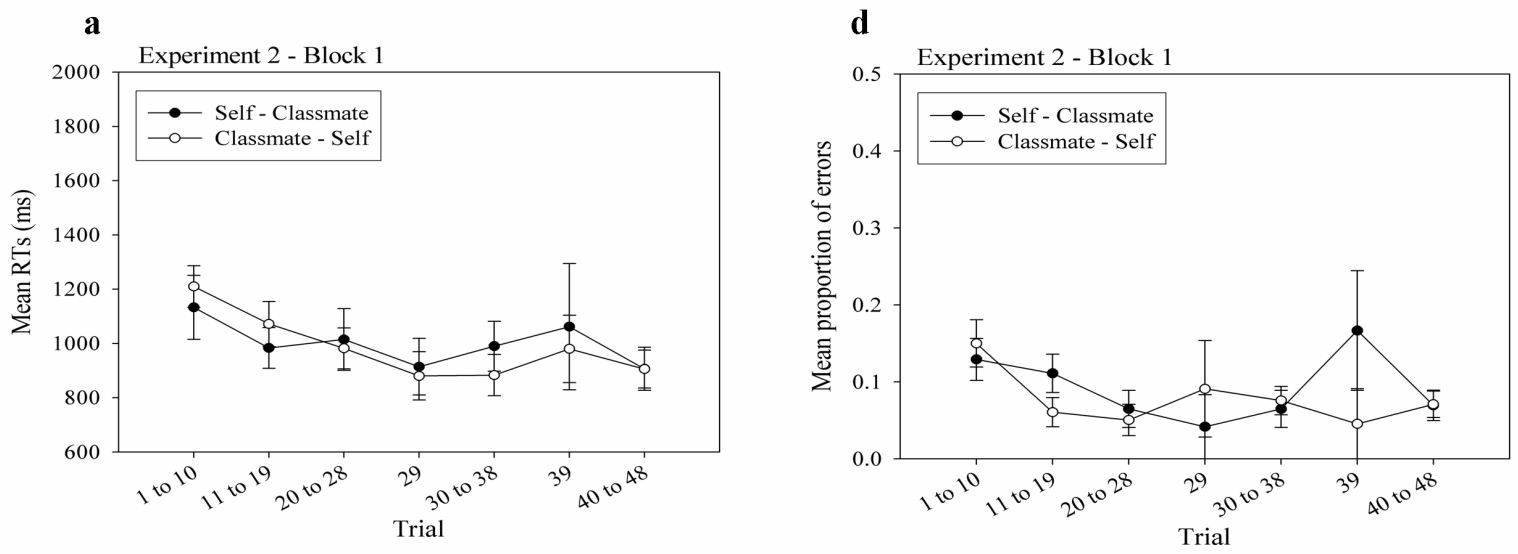

b
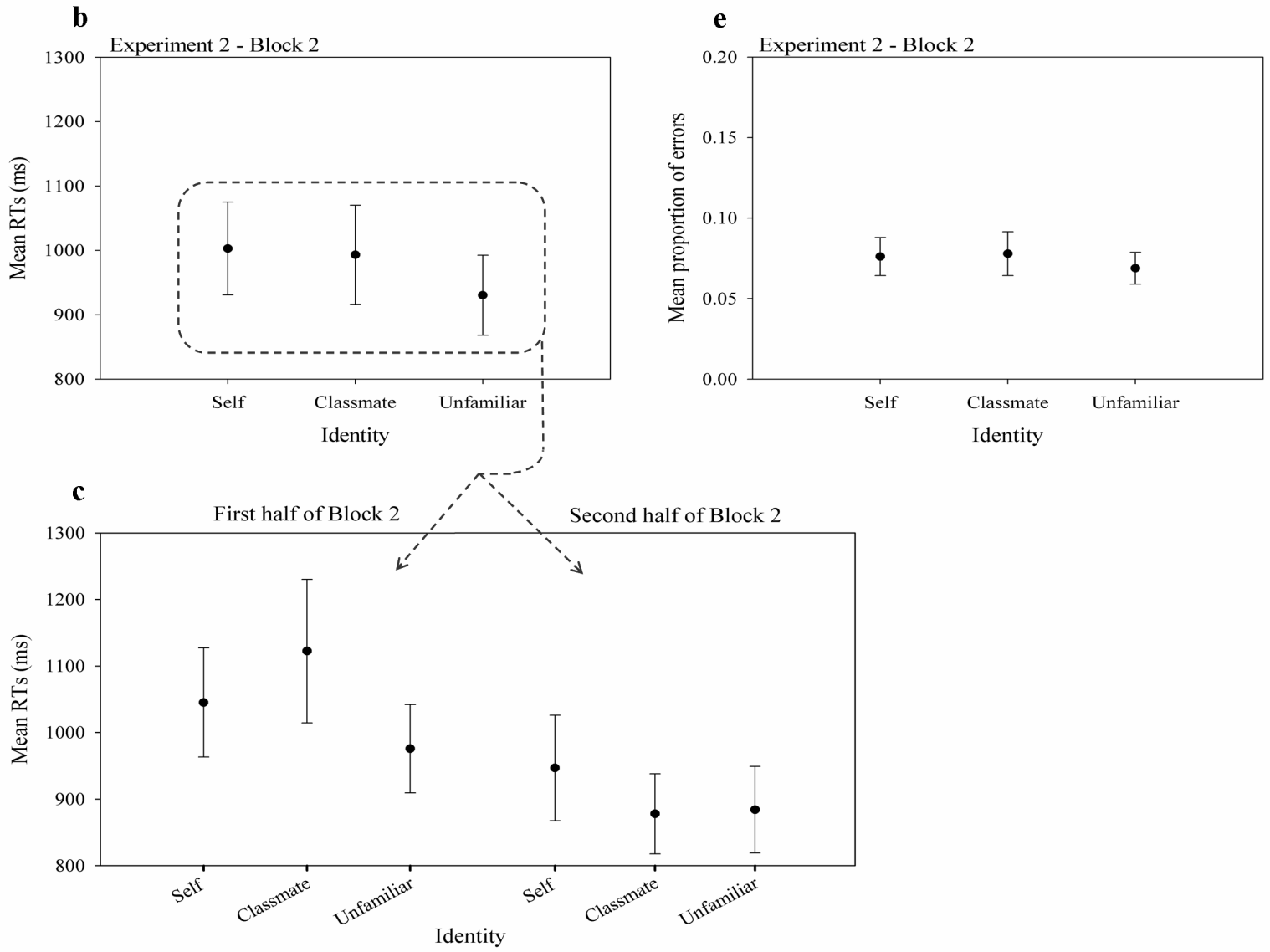

Figure 3. Results of Experiment 2: (a) Mean reaction times $(R T s)$ and $(d)$ mean error rates to the digit-parity task as a function of the order of presentation of the familiar faces in Block 1; familiar distractor faces appeared on trials 29 and 39. (b) Mean RTs and (e) mean error rates as a function of the Identity of the distractor face presented in Block 2. (c)Mean RTs as a function of the distractor face's Identity presented on the first and the second halves of Block

2 . Error bars represent the standard error of the means. 


\subsection{Results}

Data of 2 participants could not be collected entirely ( 1 because of technical reasons and 1 because the participant felt uncomfortable during the experiment) and were discarded from all analyses.

\subsubsection{Reaction times}

Data of 8 other participants who made errors on Trial 29 or 39 were discarded.

Block 1. A mixed two-way 2 (Order: self-classmate, classmate -self) by 5 (Trial: 20 to 28, 29, 30 to $38,39,40$ to 48 ) ANOVA with repeated measures on the last factor was conducted on RTs. There was no significant effect of Trial, $F(4,144)=1.02, p=0.40$, no significant effect of Order, $F(1,31)<1$, and no significant interaction, $F(4,124)<1$ (see Fig.3a).

Block 2. A one-way ANOVA with Identity (self, classmate, unfamiliar) as within-subject factor was conducted on RTs and showed no significant effect, $F(2,74)=2.28, p=0.11$ (see Fig.3b). Nonetheless, as the pattern of performance was quite similar to that observed in Block 2 of Experiment 1 (see Fig.2b and Fig.3b) we split data of Block 2 in 2 parts to examine separately the pattern of performance on the first and second halves of trials. The analysis of RTs during the first half of Block 2 revealed a marginal effect of Identity, $F(2,74)$ $=3.911, p=0.0504$. HSD Tukey post-hoc indicated that the difference between the RTs in the 'classmate's face' condition $(M=1122 \mathrm{~ms})$ and the RTs in the 'unfamiliar face' condition $(M=976 \mathrm{~ms})$ was significant, $p=0.039$. RTs in the 'self-face' condition $(M=$ $1045 \mathrm{~ms}$ ) did not differ from RTs in the 'classmate's face' condition, $p=0.39$, or in the 'unfamiliar face' condition, $p=0.47$ By contrast, the analysis of RTs during the second half of Block 2 did not show any effect of Identity, $F(2,74)=1.245, p=0.29$ (see Fig.3b).

\subsubsection{Error rates}

Block 1. A mixed two-way 2 (Order: self-classmate, classmate-self) by 5 (Trial: 20 to 28, 29, 30 to 38, 39, 40 to 48) ANOVA with repeated measures on the last factor was conducted on error rates. There was no significant effect of Trial, $F(4,176)<1$, no significant effect of Order, $F(1,44)<1$, and no interaction, $F(4,180)=1.32 ; p=0.27$ (see Fig.3d).

Block 2. A one-way ANOVA with Identity (self, classmate, unknown) as within-subject factor was conducted on error rates and showed no significant effect, $F(2,90)<1$ (see Fig.3e).

\subsection{Discussion}

In agreement with previous studies using the own name (Gronau et al., 2003), present results indicate that the self-face presented outside the focus of attention and irrelevantly for the ongoing task produces no distraction in Block 1 or in Block 2 overall, as reflected by both reaction times and error rates. However, a complementary analysis of Block 2 indicated that the facial identity marginally affected reaction times on the first half of trials. Here, the distraction was due to the processing of the classmate's face. Yet, as in Experiment 1, this effect disappeared in the second part of Block 2. This might indicate that after a few dozen of trials, when participants master the digit-parity task, they start shifting their attention at periphery and process the distractor faces. These shifts produce a weak interference effect that is not strong enough to overall affect performance in Block 2 and that habituates quickly. By contrast, in Block 1 the two appearances of the familiar faces did not interfere with the digitparity task, probably because at that time the participants' attentional resources are still devoted to the digit-parity task. This small effect of familiar faces irrelevantly presented at periphery might seems at odds with Gronau et al. (2003)'s findings. However, this effect appeared lately in the experiment, lasted a very limited number of trials and was detected only through post-hoc analyses in the absence of a main effect of Identity. More importantly the occurrence of this effect indicates that facial identity was perceivable at this eccentricity. In 
other words, the non-occurrence of interference effect in Block 1 cannot be attributed to a lack of perception of peripheral distractor faces.

\section{GENERAL DISCUSSION}

Present results indicate that the self-face, a particularly distinctive feature of the self, has no enduring distractive power compared with unfamiliar faces when it is presented at fixation, within the observer's attentional focus. Indeed, the self-face was only momentarily more distractive than unfamiliar faces. In addition, crucially the self-face was never consistently more distractive than that of another familiar person. Therefore, present results suggest that the allocation of attention was temporarily driven by the high familiarity of the to-be-ignored distractor faces rather than by the self-referential properties of the self-face. Yet, and contrary to the participant's name which may be shared by other individuals, the participant's own face is exclusively self-referential. Thus, one might have expected that it is even more likely than one's own name to attract attention by comparison with other faces. However the present study demonstrates that one's own face is not an exceptional kind of stimulus since it rapidly loses its attention grabbing character. The present study extends Harris and Pashler (2004)'s work by showing that even a unique self-referential stimulus such as one's own face is not a potent distractor, at least when its presentation is not related with the ongoing task.

Moreover, in the present study, the self-face did not elicit reliable distraction effect when it was presented outside the focus of attention. This result is consistent with Gronau et al.'s (2003) study reporting that the participant's own name did not produce any distraction when presented outside the focus of attention and irrelevantly to the ongoing task. Nonetheless, our data indicated that participants temporarily shifted their attention towards peripheral faces once they mastered the digit-parity task. Importantly, the observation that the capacity of the familiar faces to provoke a distraction was dependent upon their location within the focus of attention indicates that this distraction is not due to an automatic capture of attention (see also Bundesen et al., 1999). Indeed, in Experiment 1 the central location of the faces between the two target digits forced participants to attend to the distractor faces (despite of the instruction) in order to perceive the two digits. By contrast, in Experiment 2, there was no need to attend to the distractor faces presented at periphery in order to process the target digits. Our results indicate that in this case participants successfully followed the instruction to ignore the faces in the first part of the experiment since the presentation of the familiar faces did not affect reaction times. In the second part of the experiment however, they apparently temporarily shifted their attention towards peripheral faces. Rather, present findings suggest that familiar faces hold attention and elicit a transient difficulty to disengage attention only once they are attended $^{1}$, as in Experiment 1 and in the second part of Experiment 2 (see Fox, Russo, Bowles, \& Dutton, 2001, for similar findings with threatening words; see also Weierich, Treat, \& Hollingworth, in press, for a recent review on the distinction between capture and retention of attention in anxiety). This hypothesis should be addressed more precisely and with paradigms allowing a clear distinction between these two components of capture and retention of attention in future work. Note that the digit-parity task we used is a quite demanding task. Harris and Pashler (2004)'s study showed that the attention grabbing capacity of one's own name depended on the amount of available resources. It is possible that a less demanding task would have allowed more substantial shifts of attention towards the peripheral distractor faces. This should also be addressed in future work.

In sum, present result does not support the widespread claim that self-referential stimuli or information important to the participant automatically summon attention. Yet, such a claim is still viewed as evidence for the late selection theory of attention even in recent cognitive

\footnotetext{
${ }^{1}$ We thank Jan Theeuwes for this suggestion.
} 
psychology textbooks (e.g. Smith \& Kosslyn, 2007; Solso, MacLin, \& MacLin, 2005; Sternberg, 2006). Moreover, the present results, as well as those of Harris and Pashler (2004)'s study, stress that the response to one's own face or one's own name habituates very rapidly. This finding has important practical and clinical implications. Indeed, recent neuropsychological research used self-referential stimuli such as the patients' own name in order to assess residual awareness of the environment in non-communicative brain-damaged patients (i.e., patients in a vegetative or in a minimally conscious state). In such studies, properties of a patient's brain responses (e.g. ERPs) elicited when hearing her/his own name is supposed to inform us about the perception of this stimulus in the environment (Perrin et al., 2006; see also Holeckova, Fischer, Giard, Delpuech, \& Morlet, 2006; Perrin et al., 2005). The fact that responses to self-referential may habituate quickly has not been addressed in such studies, presumably because, again, it was assumed that self-referential materials automatically grab attention (e.g. Holeckova et al., 2006; Sui et al., 2006; but see Laureys, Perrin, \& Brédart, 2007). Yet, in most of these studies, the same self-referential stimulus was usually presented several times during the experimental session. After the results from Harris and Pasher (2004) and the present study, it seems that averaging across repeated trials is likely to fail giving rise to patients' responses that occurred after the first few presentations of selfreferential materials.

In conclusion, present findings demonstrate that a unique and distinctive self-referential stimulus such as one's own face is not a potent distractor compared to other faces. The distraction it produces does not differ from that produced by another familiar face, is only temporary and is modulated by the position of the face within the participant's focus of attention. Future work should clarify whether this distraction is due to a difficulty to disengage attention as hypothesized here and/or whether the self-face has the capacity to automatically capture attention in some conditions.

\section{Acknowledgments}

This research was supported by a grant from the Belgian Fonds de la Recherche Fondamentale Collective (Grant $n^{\circ} 8.4506 .05-2.4539 .05$ ) to SB. CD is Scientific Research Worker at the National Fund for Scientific Research (Belgium). We warmly thank Nathalie Jamaer for her help with preparation of participants' pictures and data collection. We thank Asher Cohen, Johan Wagemans and an anonymous reviewer for their constructive comments. 


\section{REFERENCES}

Arnell, K.M., Shapiro, K.L., \& Sorensen, R.E. (1999). Reduced repetition blindness for one's own name. Visual Cognition, 6, 609-635.

Brédart, S., Delchambre, M., \& Laureys, S. (2006). One's own face is hard to ignore. The Quarterly Journal of Experimental Psychology, 59, 46-52.

Broadbent, D.E. (1958). Perception and Communication. London: Pergamon Press.

Bundesen, C., Kyllingsbaek, S., Houmann, K. J., \& Jensen, R. M. (1997). Is visual attention automatically attracted by one's own name? Perception \& Psychophysics, 59, 714720.

Deutsch, J., \& Deutsch, D. (1963). Attention: Some theoretical consideration. Psychological Review, 87, 272-300.

Fox, E., Russo, R., Bowles, R., \& Dutton, K. (2001). Do threatening stimuli draw or hold visual attention in sub-clinical anxiety? Journal of Experimental Psychology: General, 130, 681-700.

Gray, H. M., Ambady, N., Lowenthal, W. T., \& Deldin, P. (2004). P300 as an index of attention to self-relevant stimuli. Journal of Experimental Social Psychology, 40, 216224.

Gronau, N., Cohen, A., \& Ben-Shakhar, G. (2003). Dissociations of personally significant and task-relevant distractors inside and outside the focus of attention: A combined behavioral and psychophysiological study. Journal of Experimental Psychology: General, 132, 512-529.

Harris, C.R., \& Pashler, H. (2004). Attention and the processing of emotional words and names: Not so special after all. Psychological Science, 15, 171-178.

Harris, C.R., Pashler, H.E., \& Coburn, N. (2004). Moray revisited: High-priority affective stimuli and visual search. The Quarterly Journal of Experimental Psychology, 57A, 131.

Holeckova, I., Fischer, C., Giard, M.-H., Delpuech, C., \& Morlet, D. (2006). Brain responses to a subject's own name uttered by a familiar voice. Brain Research, 1082, 142-152.

Kawahara, J., \& Yamada, Y. (2004). Does one's name attract visual attention? Visual Cognition, 11, 997-1017.

Laarni, J., Koljonen, M., Kuistio, A.M., Kyröläinen, S., Lempiäinen, J., \& Lepistö, T. (2000). Images of a familiar face do not capture attention under conditions of inattention. Perceptual and Motor Skills, 90, 1216-1218.

Lachter, J., Forster, K., \& Ruthruff, E. (2004). Forty-five years after Broadbent (1958): Still no identification without attention. Psychological Review, 111, 880-913.

Laureys, S., Perrin, F., \& Brédart, S. (2007). Self-consciousness in non-communicative patients. Consciousness and Cognition, 16, 722-741.

Mack, A., \& Rock, I. (1998). Inattentional blindness. Cambridge, MA: MIT Press.

Moray, N. (1959). Attention in dichotic listening: Affective cues and the influence of instructions. Quarterly Journal of Experimental Psychology, 11, 56-60.

Ninomiya, H., Onitsuka, T., Chen, C.H., Sato, E., \& Tashiro, N. (1998). P300 in response to the subject's own face. Psychiatry and Clinical Neurosciences, 52, 519-522.

Perrin, F., Maquet, P., Peigneux, P., Ruby, P., Degueldre, C., Balteau, E., Del Fiore, G., Moonen, G., Luxen, A., \& Laureys, S. (2005). Neural mechanisms involved in the detection of our first name: A combined ERPs and PET study. Neuropsychologia, 43, 12-19.

Perrin, F., Schnakers, C., Schabus, M., Degueldre, C., Goldman, S., Brédart, S., Faymonville, M.-E., Lamy, M., Moonen, G., Luxen, A., Maquet, P., \& Laureys, S. (2006). Brain response to one's own name in vegetative state, minimally conscious state, and lockedin syndrome. Archives of Neurology, 63, 562-569. 
Shapiro, K.L, Caldwell, J., \& Sorensen, R.E. (1997). Personal names and the attentional blink: A visual "cocktail party" effect. Journal of Experimental Psychology: Human Perception and Performance, 23, 504-514.

Smith, E.E., \& Kosslyn, S.M. (2007). Cognitive Psychology: Mind and Brain. Upper Saddle River, New Jersey: Prentice Hall.

Solso, R.L, Maclin, M.K \& Maclin, O.H (2005). Cognitive Psychology (7th ed.). Boston: Pearson Education.

Sternberg, R.J. (2006). Cognitive Psychology (4th ed.).. Belmont, CA: Thomson Wadsworth.

Sugiura, M., Kawashima, R., Nakamura, K., Okada, K., Kato, T., Nakamura, A., Hatano, K., Itoh, K., Kojima, S., \& Fukuda, H. (2000). Passive and active recognition of one's own face. NeuroImage, 11, 36-48.

Sui, J., Zhu, Y., \& Han, S. (2006). Self-face recognition in attended and unattended conditions: An event-related brain potential study. NeuroReport, 17, 423-427.

Tanaka, J.W., Curran, T., Porterfield, A.L., \& Collins, D. (2006). Activation of preexisting and acquired face representations: The N250 event-related potential as an index of face familiarity. Journal of Cognitive Neuroscience, 18, 1488-1497.

Tong, F., \& Nakayama, K. (1999). Robust representations for faces: Evidence from visual search. Journal of Experimental Psychology: Human Perception and Performance, 25, 1016-1035.

Weierich, M.R, Treat, T.A., \& Hollingworth, A. (in press). Theories and measurement of visual attentional processing in anxiety. Cognition \& Emotion.

Wolford, G., \& Morrison, F. (1980). Processing of unattended visual information. Memory \& Cognition, 8, 521-527. 